\title{
Unsteady aerodynamic validation experiences from the Aeroelastic Prediction Workshop
}

\author{
Jennifer Heeg ãnd Pawel Chwalowski, ${ }^{\dagger}$ \\ NASA Langley Research Center, Hampton, VA 23681-2199
}

\begin{abstract}
The AIAA Aeroelastic Prediction Workshop (AePW) was held in April 2012, bringing together communities of aeroelasticians, computational fluid dynamicists and experimentalists. The extended objective was to assess the state of the art in computational aeroelastic methods as practical tools for the prediction of static and dynamic aeroelastic phenomena. As a step in this process, workshop participants analyzed unsteady aerodynamic and weakly-coupled aeroelastic cases. Forced oscillation and unforced system experiments and computations have been compared for three configurations. This paper emphasizes interpretation of the experimental data, computational results and their comparisons from the perspective of validation of unsteady system predictions. The issues examined in detail are variability introduced by input choices for the computations, post-processing, and static aeroelastic modeling. The final issue addressed is interpreting unsteady information that is present in experimental data that is assumed to be steady, and the resulting consequences on the comparison data sets.
\end{abstract}

\section{Nomenclature}

$\begin{array}{ll}C_{M} & \text { Pitching moment coefficient } \\ C_{p} & \text { Coefficient of pressure } \\ \mathrm{f} & \text { Frequency, Hz } \\ M & \text { Mach number } \\ \mathrm{x} / \mathrm{c} & \text { Normalized chord location, chordwise coordinate/local chord length } \\ y^{+} & \text {Dimensionless, sublayer-scaled wall coordinate of first node away from surface } \\ \alpha & \text { Angle of attack } \\ \eta & \text { Normalized span location, spanwise coordinate/semi-span } \\ \text { AePW } & \text { Aeroelastic Prediction Workshop } \\ \text { BSCW } & \text { Benchmark Supercritical Wing } \\ \text { CAE } & \text { Computational Aeroelasticity } \\ \text { CFD } & \text { Computational Fluid Dynamics } \\ \text { CRM } & \text { Common Research Model } \\ \text { ETW } & \text { European Transonic Windtunnel } \\ \text { FRF } & \text { Frequency Response Function } \\ \text { HIRENASD } & \text { HIgh REynolds Number AeroStructural Dynamics } \\ \text { RSW } & \text { Rectangular Supercritical Wing } \\ \text { TDT } & \text { Transonic Dynamics Tunnel }\end{array}$

\section{Introduction}

CREDIBILITY of computational methods has improved in recent years, in large part due to dedicated verification and validation efforts. Relying on definitions established by the Department of Energy ${ }^{1,2}$ and the AIAA: ${ }^{3-5}$ validation is the process of determining how well the results from a computational model compare with the characteristics of the physical system of interest.

\footnotetext{
* Senior Research Engineer, Aeroelasticity Branch, MS 340, Senior Member AIAA

${ }^{\dagger}$ Senior Research Engineer, Aeroelasticity Branch, MS 340, Senior Member AIAA
} 
Participants in the Drag Prediction Workshop ${ }^{6,7}$ (DPW) series and the High Lift Prediction Workshop ${ }^{8,9}$ have performed quantitative and qualitative assessments of a significant cross-section of computational methods, relative to experimental data. These efforts have been used to determine the level of confidence that can be placed in computational results, focusing on steady state rigid configurations. The Aeroelastic Prediction Workshop (AePW) has been crafted to follow in the footsteps of these prior workshops, extending benchmarking efforts to unsteady computational methods and coupled fluid/structure methods

The AIAA Aeroelastic Prediction Workshop was held in conjunction with the 53rd AIAA Structures, Structural Dynamics, and Materials Conference on April 21-22, 2012, in Honolulu, Hawaii. The AePW and its follow-on activities are collaborations within the aeroelastic community to assess the state-of-the-art in computational aeroelasticity (CAE), assess the experimental data available for performing this assessment and provide a roadmap forward. The AePW was not a strict validation activity, however, the organizing committee drew on the logic and process that guides validation efforts, dividing the complex problem of nonlinear unsteady aeroelastic analysis of an aerospace vehicle into simpler components. The components, or building blocks, were formulated to focus on specific aspects of the underlying physics. The coarse-grain building blocks in aeroelasticity are: 1) unsteady aerodynamics; 2) structural dynamics; and 3) coupling between the fluid and the structure. The AePW organizing committee members viewed the unsteady aerodynamics portion of the problem as the most challenging and the aspect that introduced the most uncertainty into an aeroelastic analysis. The 2012 workshop focused primarily on validating unsteady aerodynamic models and methods, with an initial venture into a weakly coupled aeroelastic system.

The approach taken was to utilize existing experimental data sets in the building-block approach, incrementally validating targeted aspects of CAE tools. Two types of experimental data were used: data obtained with the model at a stabilized constant test condition and data obtained while the model was undergoing forced sinusoidal oscillations. While the focus was on forced oscillation data, it seemed a logical stepping stone in the process to include corresponding unforced - steady, stabilized — cases. The computational community was challenged to perform both steady and unsteady aerodynamic analyses on three configurations in manners that would match the results from these experimental data sets and present their results at the workshop. Three configurations served as test cases for the AePW. Each are shown mounted in their wind tunnel test configurations in figure 1: the Rectangular Supercritical Wing (RSW); the Benchmark SuperCritical Wing (BSCW) and the High Reynolds Number Aero-Structural Dynamics (HIRENASD) model.

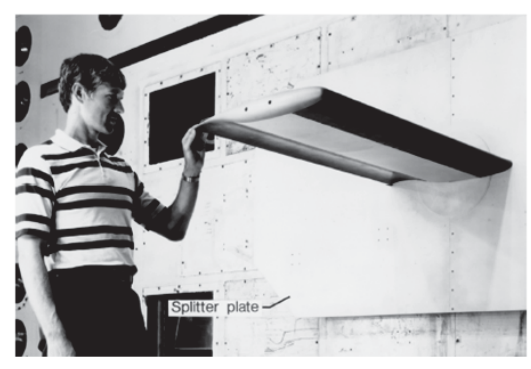

(a) RSW, mounted in TDT.

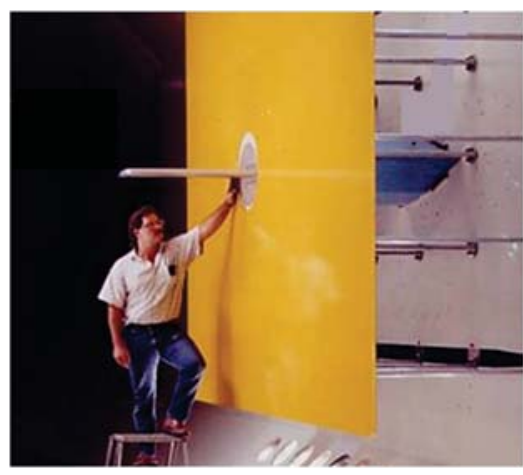

(b) BSCW, mounted in TDT.

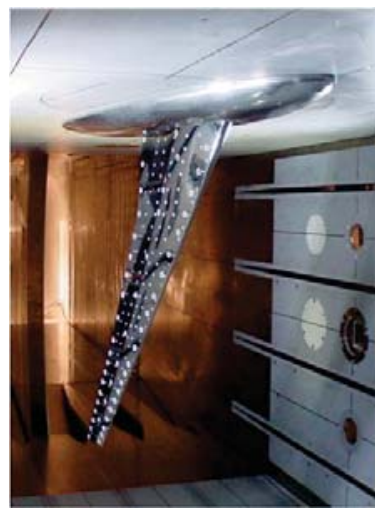

(c) HIRENASD, mounted in ETW.

Figure 1. Test configurations, shown mounted in the wind tunnels.

The RSW ${ }^{10-12}$ was tested in 1982 in the NASA Transonic Dynamics Tunnel (TDT) ${ }^{13,14}$ using Freon R-12 as the test medium. For this experiment, a rectangular planform, "rigid" supercritical wing was sidewall-mounted to a small splitter plate and oscillated in pitch, exhibiting a moderate shock and boundary-layer interaction. A summary of the AePW results for this configuration, experimental data, configuration details and additional reference material was published in 2012. ${ }^{15}$

The $\mathrm{BSCW}^{16,17}$ was also tested in the TDT, using a similar heavy gas (R-134a) as the test medium. This data set was acquired in 2000 for a "rigid" rectangular planform similar to the RSW that was sidewall-mounted to a large splitter plate assembly and oscillated in pitch via the TDT Oscillating Turntable,$^{18}$ exhibiting a strong shock and boundary-layer-induced separated flow at a moderate angle of attack. Analyses of the experimental data set can be found in Piatak and Cleckner ${ }^{18}$ and Heeg and Piatak. ${ }^{19}$ 
The HIRENASD testing, led by Rheinisch-Westfaelische Technische Hochschule (RWTH) Aachen was conducted in the European Transonic Windtunnel (ETW) in 2006, with gaseous Nitrogen as the test medium. For this experiment, a model with well-separated structural dynamic modes was mounted to the tunnel ceiling and oscillated at or near the frequency of the first bending mode, the second bending mode, or the first torsion mode. Wind tunnel model descriptions, testing and experimental data are reported in numerous publications. ${ }^{20-22}$ Numerous computational studies of HIRENASD were performed prior to using this configuration for the AePW; ${ }^{23-26}$ computational results from several of the analysts participating in the AePW have also been published. ${ }^{27-30}$

The intended build-up of complexity was undermined by the choices made in test conditions. Transonic conditions were intentionally chosen for several important reasons. Transonic conditions are often considered to be the most critical conditions with regard to aeroelastic phenomena such as flutter onset, buffet and limit cycle oscillations. ${ }^{31-33}$ In the transonic range, various flow phenomena can initiate and produce severe aeroelastic issues. As such, the most significant disagreements among computational results and between experiments and computations are observed. Coupling the criticality and the historical discrepancies drew the organizing committee to consider transonic predictions as the necessary starting point for discussion of workshop configurations and cases. In making the choice to examine transonic flow, however, simplicity was undermined. Benchmarking first against a benign subsonic test condition seems an obvious requirement in retrospect. For the HIRENASD case, a subsonic condition was added to the matrix of analysis conditions, although somewhat late in the workshop execution timeline.

Forced oscillatory excitations were chosen as the primary test data for the AePW. This type of test data has historically been used in unsteady aerodynamic validation efforts within the field of aeroelasticity. This choice of excitation relates to the aeroelastic foundation of the AePW. Experimentally, it is common for data at an instability to be dominated by an unstable (positive damping) sinusoid. An aeroelastic system is one where the aerodynamic forces and the structural deformations feed off of each other and the phasing between the forces and deformations is critical. Forced oscillatory excitation allows for straightforward examination of the phase between the forces and the deformations. Linear aeroelastic analyses are usually performed in the frequency domain and knowledge of the behavior of the system as a function of oscillation frequency has historically provided comparison data that corresponds with the computational results. This type of forcing function also provides inputs of sufficient amplitude to make the response more observable. For each unsteady test and analysis condition, the unforced system case — steady - was also analyzed and compared with corresponding experimental data. Detailed tables of all of the workshop test conditions are published on the AePW website ${ }^{34}$ and in prior publications. ${ }^{35}$

Much of the data discussed in the current paper has been presented previously and many of the lessons learned discussed in the prior publications. ${ }^{35-37}$ The interested reader is referred to these publications to obtain a more thorough understanding of the workshop participation and results. The current paper highlights a few aspects of the workshop pertinent to conducting a validation exercise in unsteady aerodynamics. The remainder of this paper is structured to first summarize the workshop discussions and findings. Four validation aspects of the workshop are then discussed in sequence: the influence of tightening the analysis variables; postprocessing effects; inclusion of aeroelastic coupling; and representations of unsteady data. The concluding sections briefly discuss recommendations for improved validation efforts and follow-on activities.

\section{Workshop summary}

The main content of this paper is devoted to validation-relevant experiences from the AePW. To provide background and context for those discussions, a summary workshop is presented here. The fundamental questions that the workshop was established to answer are:

- How good are our tools and processes, and what aspects of those tools need further development?

- How good are our experimental data bases relative to what constitutes a "good validation data set" for computational aeroelasticity.

Independent teams of analysts performed computations and supplied their results to help address these questions. Each team was free to choose their own solution methods, input parameters for those solutions, and aerodynamic grids. The grids were required to comply with an established set of gridding guidelines ${ }^{38}$ or the analysts could choose from among grids supplied by the organizing committee. Most teams chose to generate their own grids. For the aerodynamic flow solutions, the AePW teams generally chose Reynolds-averaged Navier-Stokes (RANS) flow solvers, and the majority chose to use either a Spalart-Allmaras turbulence model ${ }^{39}$ or Menter's Shear Stress Transport turbulence model. ${ }^{40}$ These choices reflect the state-of-the-art or perhaps, better-phrased, the state-of-the-current practices within the CFD community. In terms of common practice for aeroelastic solutions, this represents the practices of those on the leading edge of modeling complexity. This level of flow solution is perhaps becoming more common, however, linear 
methods such as doublet lattice aerodynamics ${ }^{41}$ and ZONA ${ }^{42}$ are still more commonly used by practitioners. Heeg et al. ${ }^{35}$ provides tables showing the analysis teams, configurations analyzed and the details of the solution methods utilized by each team.

One of the primary illustrations of this workshop was the dominance of upper surface shock motion in the system responses for transonic conditions. The principal test conditions for each configuration contained an oscillating upper surface shock as illustrated by the example results for the HIRENASD configuration in figure 2(a). The largest magnitude of the dynamics, i.e. in the Frequency Response Functions (FRFs), is the shock oscillation. For the forced oscillation cases, the shocks responded primarily at the forced motion frequency, illustrated for HIRENASD; figure 2(b) shows the magnitude of the FRF and figure 2(c) shows the phase of the FRF.

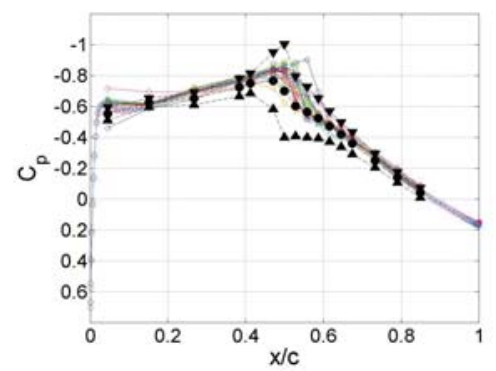

(a) Upper surface pressure coefficient distribution.

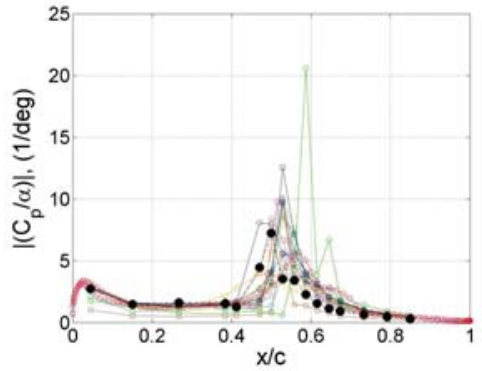

(b) Magnitude of FRF.

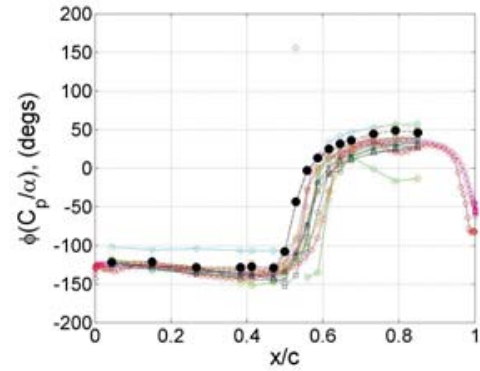

(c) Phase of FRF.

Figure 2. HIRENASD example results, Mach 0.8, $\alpha=1.5^{\circ}, \mathrm{s}, \eta=0.59$ (Station 4), 59\% span (Black: Experiment; Colors: Mean value distributions from computations).

Another illustration of workshop results defines the flow regions where the RANS solutions appear to be satisfactory when compared to experimental data. In cases without large separated flow regions or significant wind tunnel wall boundary layer effects, the RANS computational methods capture qualitative features for these fairly thick supercritical airfoils, keeping with previous historical findings. ${ }^{43}$ The scatter among the AePW results is large where viscous effects are significant. In the cases where separated flow or geometrically-thickened boundary layers are indicated by the experimental data, these methods appear to qualitatively mis-predict both the steady pressure distributions and the unsteady pressure responses. These points are illustrated using the unforced system pressure distributions for the RSW (figure 3(a)) and the BSCW (figure 3(b)). The regions where the computational results qualitatively differ from the experimental data include the separated flow region aft of the BSCW upper surface shock and the lower surfaces in the supercritical airfoil cusp regions at the aft end of each airfoil. The mis-predictions are thought to be due to the time-averaging introduced through the turbulence models employed in the RANS and URANS solvers. Even in a time-accurate simulation, if the time step is not small enough, or there are not enough subiterations, vorticity and separation features are smeared, and reattachment in particular is missed. ${ }^{44}$

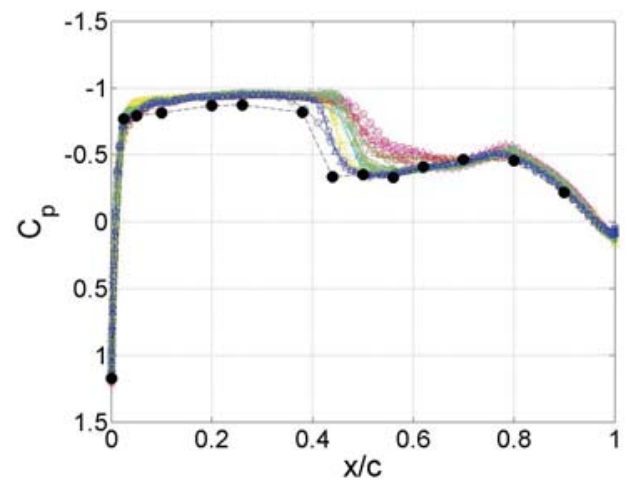

(a) RSW, station $2,59 \%$ span $\left(\alpha=2^{\circ}, \mathrm{M}=0.825\right)$.

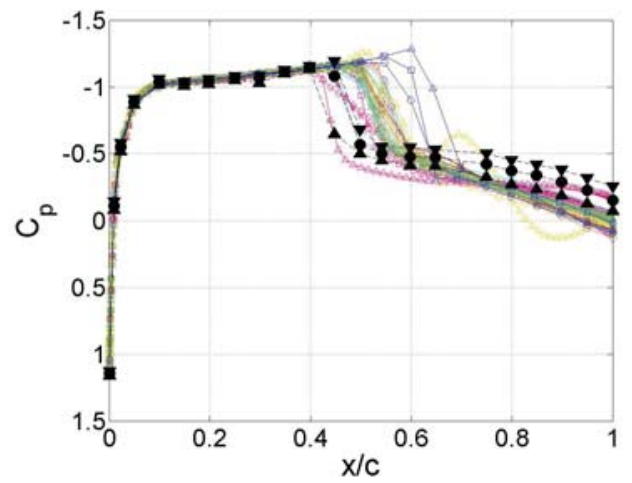

(b) $\operatorname{BSCW}, 60 \% \operatorname{span}\left(\alpha=5^{\circ}, \mathrm{M}=0.85\right)$.

Figure 3. Upper surface pressure coefficient distributions, unforced system data (Black: experiment mean or mode, maxima and minima; Colors: Computational mean values).

Examination of each configuration offered different lessons learned. A few of those are summarized here. 
- The most challenging aspect of analyzing the RSW configuration was introduced by poor experiment set-up. The influences of the proximity of the model to the wind tunnel wall and the undersized splitter plate were not as fully understood when the model was originally tested as they are today. The consequence of attempting to capture the wall influences was that the CFD solutions varied widely, even for the unforced system results. The variation present in these results is not viewed as an accurate assessment of the state of the art, but rather as an indicator of the variation introduced by analysts' input choices and possible misinterpretations of normalization parameters. ${ }^{15}$

- Shock-induced separated flow and trailing edge separation was present for the BSCW configuration at our selected test conditions. Lower surface separation in the cusp region was also likely to have occurred. The computational methods that were applied had difficulty producing converged solutions for the unforced system and for the lower frequency forced oscillation case. The convergence problems of these solutions are attributable to the complexity of the flow field and lack of appropriate fidelity in the turbulence models. ${ }^{19,45}$

- HIRENASD was not as challenging as the simpler geometries of the RSW and BSCW due to test condition selection and airfoil geometry. Taken as a whole, the HIRENASD computational results compared better with experimental data than the other two configurations, attributable to the difference in flow physics. Specifically, the weaker shocks and attached flow were more easily captured by the flow solvers chosen. The qualitative differences in the flow field are assessed to be due to the less severe airfoil geometry, Mach number and angle of attack. ${ }^{36}$ One of the analysis conditions was a zero-lift case, chosen with the thought that the shock would be less stationary and would prove more difficult to correctly predict. Because no case contained a truly stationary shock - as will be discussed later in this paper- the zero-lift case turned out to be less of a challenge to analysts than the test case with a strong upper surface shock. ${ }^{46,47}$ One aspect of CAE analysis that HIRENASD analyses illustrated is that to correctly compute the steady pressure distribution, it is important to assess the rigidity of the model and obtain the correct deformed shape for flexible models. Failure to do this was demonstrated to result in effective changes in the local chordwise angle of attack. Using the rigid shape, rather than the deflected aeroelastic shape resulted in overprediction of the pressure distribution. ${ }^{29}$ The impact on the forced oscillation results is discussed in Pranata et al. ${ }^{47}$

The workshop also produced some discussions and lessons that are fairly universal to successfully performing this type of workshop. A few of the most important are summarized below.

- A workshop goal was to determine the relative significance of computational choices. Large variations were observed within the computational results submitted for both the unforced system response and the frequency response functions. There were insufficient statistical sample sizes to assess the causes from among the possible sources. That is, not enough computational data sets were submitted with consistent parameters to differentiate one factor from another. Differences in grid, time step size, convergence level, turbulence model and other numerical specifications exist among the submitted results. ${ }^{15}$

- Computational solutions were not run, typically, for a sufficient amount of time to utilize classical techniques for assessing and reducing the errors in Fourier coefficients, which are used in computing the frequency response functions. This is not a fundamental limitation, but rather a choice made by the computational teams, either by oversight or due to computational expense. When the frequency of the response is not exactly known or not exactly captured in an integer number of data samples, this becomes important. For the HIRENASD case, sufficient time records were generated by several analysis teams to determine that for the HIRENASD cases, the responses were linear relative to the forcing function at the frequency of oscillation. Under those circumstances, a single complete cycle of response is sufficient. Without the extended-time analyses, there would have been zero confidence in the single-cycle FRF results. ${ }^{48}$ For the separated flow case (BSCW configuration), the record length requirement with higher fidelity methods is the subject of continuing investigation. ${ }^{45}$

- The data processing for CFD data is significantly different from classical experimental data processing. It is much more reminiscent of processing signals generated from a multisine signal. The results are highly sensitive to exactly capturing single cycles and setting Fourier analysis time record length to match. ${ }^{4-50}$ Classical Fourier analysis techniques may not be sufficient for analysis of CFD data that consists of limited sample sizes and short time records. New techniques that can be equally applied to both CFD and experimental data should be investigated.

The workshop effort also illuminated numerous orgnanizational issues. While many of these are discussed in the referenced documents, the following seems important to future validation-type activities. A computationallyrelevant data set is best generated if the people formulating the computations also play major roles in formulating and conducting the experiment. Said the other way, it is equally valid. Applying an experimental data set to validate a 
computational method is best done if those who formulate and conduct the experiment play major roles in formulating the computations. This same mindset is useful with regard to working across fields to understand the flow physics, and develop and apply computational methods. This is exemplified within the AePW effort, where there is an unmet requirement to understand more about the boundary layer flow physics associated with separated flow fields and their proper modeling.

From the lessons learned during the workshop, four specific aspects that pertain to conducting a validation exercise were explored. These four aspects are discussed in detail in the remainder of this paper.

\section{Validation aspect \#1: Tightening the analysis variables}

Nineteen analysis teams provided computational results for the workshop. The majority of the AePW workshop participants built their own grids. Specifically for the HIRENASD configuration, only three out of 14 analyses teams used grids provided by other AePW teams. Participants conducted analyses using their CFD software with their choice of turbulence model, number of mode shapes in fluid-structure coupling analysis, and they post-processed their dynamic data using their own software to calculate the frequency response functions of pressure due to reference displacement. The choice of computational methods, including flow solvers, associated turbulence model and flux construction selections are detailed in reference. ${ }^{35}$ The majority of the analysis teams utilzed Reynolds Averaged Navier-Stokes (RANS) flow solvers. RANS equations are time-averaged equations of motion for fluid flow. The timeaveraged assumptions involve separation of chaotic velocity fluctions from the mean flow velocity. This in turn requires the use of a turbulence model which is usually tuned for a specific flow physics situation. The unforced system analyses were in general performed by converging RANS solutions to steady state. The forced oscillation simulations were performed using unsteady RANS (URANS) codes, solved in a time-accurate manner with subiterations to converge the solution at each of the time steps. Two HIRENASD analysis teams did not use RANS flow solvers. One team employed Euler flow solutions and one team performed full-potential flow solutions. One of the primary conclusions from the workshop was that too many variables existed among the solutions. This corresponded to a conclusion from the first three drag prediction workshops, as stated by Morrison and Hemsch, ${ }^{51}$ "After three workshops, it is still clear that grids remain a first order effect and obtaining high quality grids is the first step to obtaining a high quality solution."

Recognizing that these factors likely contributed significantly to the scatter observed within the results, a subset of the HIRENASD analysts chose to revisit the configuration and attempt to eliminate these variables. A common grid study was conducted using four Reynolds-averaged Navier-Stokes (RANS) flow solvers: TAU ${ }^{52,53}$ from DLR, Edge ${ }^{54}$ from FOI, Fluent ${ }^{55}$ from ANSYS, and FUN3D ${ }^{56}$ from NASA. The results of HIRENASD configuration re-analysis using a common grid approach and the same turbulence model, specifically the Spalart-Allmaras one equation model ${ }^{39}$ were presented in detail at the 2013 IFASD meeting. ${ }^{48}$

SOLUTION PROCESS The fluid-structure coupling in the TAU, Edge, and FUN3D software is accomplished by modal structural solvers. The modal structural solvers each utilized 30 mode shapes in fluid-structure coupling analysis. This method requires the modes to be interpolated onto CFD surface mesh. To eliminate another source of differences among the computational results, the modes projected at DLR were used in the TAU, Edge and FUN3D computations. The first 30 modes were projected onto the CFD mesh including wing and fuselage. Here, the mode shape values were set to zero on the fuselage, with the exception of a very narrow region near the wing and the fuselage junction. This process eliminated a discontinuity in mode shape values at this junction, which had caused flow solvers to abort. Note that the Fluent solver used its own internal method of coupling fluid and structure for aeroelastic solutions. Additionally, the dynamic results were post-processed using the same software to produce the frequency response functions.

GEOMETRY AND GRIDS Based on an IGES definition of the geometry, common grids were built with the hybrid, quad-dominated grid generation software Solar. ${ }^{57}$ The advantage of using unstructured quadrilateral elements, rather than triangular elements on the wing and fuselage surfaces, is the drastic reduction in the number of cells required in the overall computational domain for comparable grid density. The flow domain was discretized using a hybrid grid composed of hexahedrons, prisms, tetrahedrons and pyramids. Regions in the HIRENASD geometry where particular attention must be paid to the grid generation with respect to the expected flow conditions can be identified as follows:

- Wing-fuselage junction, in particular near the trailing edge

- Blunt trailing edge 
- Wingtip near leading and trailing edge

- Wing upper surface for shock resolution

The final CFD grids used for both the steady and unsteady simulations are characterized by the properties listed in Table 1.

Table 1. Solar grid statistics for HIRENASD configuration.

\begin{tabular}{l|c|c|c}
\hline \hline & Coarse Mesh & Medium Mesh & Fine Mesh \\
\hline Number of nodes & $1,034,003$ & $2,448,805$ & $7,206,319$ \\
Number of elements & $1,530,645$ & $4,003,410$ & $13,169,981$ \\
Hexahedra & 907,276 & $2,087,562$ & $5,892,524$ \\
Tetrahedra & 593,662 & $1,858,259$ & $7,126,948$ \\
Wedges & 8,554 & 10,128 & 16,588 \\
Pyramids & 21,153 & 47,461 & 133,921 \\
First grid point @ wall, (meter) & $4.4 e^{-07}$ & $2.9 e^{-07}$ & $2.0 e^{-07}$ \\
& $\left(y^{+}=1.00\right)$ & $\left(y^{+}=0.66\right)$ & $\left(y^{+}=0.44\right)$ \\
Wind-Tunnel Ceiling Boundary Cond. & Symmetry & Symmetry & Symmetry \\
\hline \hline
\end{tabular}

STATIC AEROELASTIC COMPUTATIONAL RESUlts The aeroelastic solution process requires that the rigid body steady solutions be obtained first. These solutions are then used as initial conditions for the corresponding static aeroelastic solutions. The aerodynamic coefficients were obtained from both the rigid body and the static aeroelastic calculations for the coarse, medium, and fine grids. An example of these results- pitching moment coefficient of the static aeroelastic solution- is plotted as a function of grid factor in figure 4. The ' $N$ ' in this plot represents number of grid points. In this figure, grey diamonds show values submitted to the AePW. The colored symbols and lines show those obtained in the common grid post-workshop computations. These results show that the solutions from four solvers converge in the direction of a common value. They are also more tightly clustered than the AePW values for the same grid size. An additional finer grid(s) study is necessary to determine further trends in solution dependence on grid refinement.

The pitching moment coefficient was chosen for examination here because it represents the least amount of improvement in the computational results scatter, and it combines the shock strength and location into one quantity. Those results that have a higher pitching moment coefficient are tending downward with increasing grid size and those that have a lower pitching moment coefficient are tending upward with increasing grid size. This was not an observable trend in the AePW results.

The pressure coefficient distributions were compared in the same way as in figure 2 for the AePW results. $C_{p}$ results for the upper surface at the 59\% span location (station 4) are presented in figure 5(a) and compared with the experimental data, zoomed in to the shock region. In this figure, colored lines and symbols represent the computational data produced in the common grid study, with a unique color for each mesh density and a unique symbol for each of the flow solvers. The background grey lines represent the 24 data sets submitted by the AePW participants. Yellow circles represent the statistical mode of the experimental data, which is the value that appears most often in the experimental set of data. The yellow triangular symbols show the maximum and minimum bounds of the experimental data. It is clear from these figures that the post-workshop analyses — with common grid and the same turbulence model — bring the computational data closer together when compared to AePW computational results database. Several conclusions can be drawn from these figures: (1) None of the flow solver results perfectly match the experimental data, (2) The medium and fine grid solutions are tightly grouped on the upper surface and are closer to the experimental data than the coarse grid solution, (3) there is a large scatter in results on the upper surface across the shock region; however, the medium and fine grid solutions more closely resemble the experimental shock shape than the coarse grid solution.

FORCED EXCITATION COMPUTATIONAL RESULTS Forced excitation analyses were performed in the common grid study using the TAU, Edge, and FUN3D flow solvers. Numerically, the motion of the wing was accomplished via modal excitation at $78.9 \mathrm{~Hz}$. The unsteady simulations were performed by restarting computations from the static aeroelastic solutions. Typically, two to four cycles of solutions are run before the surface pressure data on the entire wing are collected at each time step. After the surface pressure data were collected, each participant in this study used 


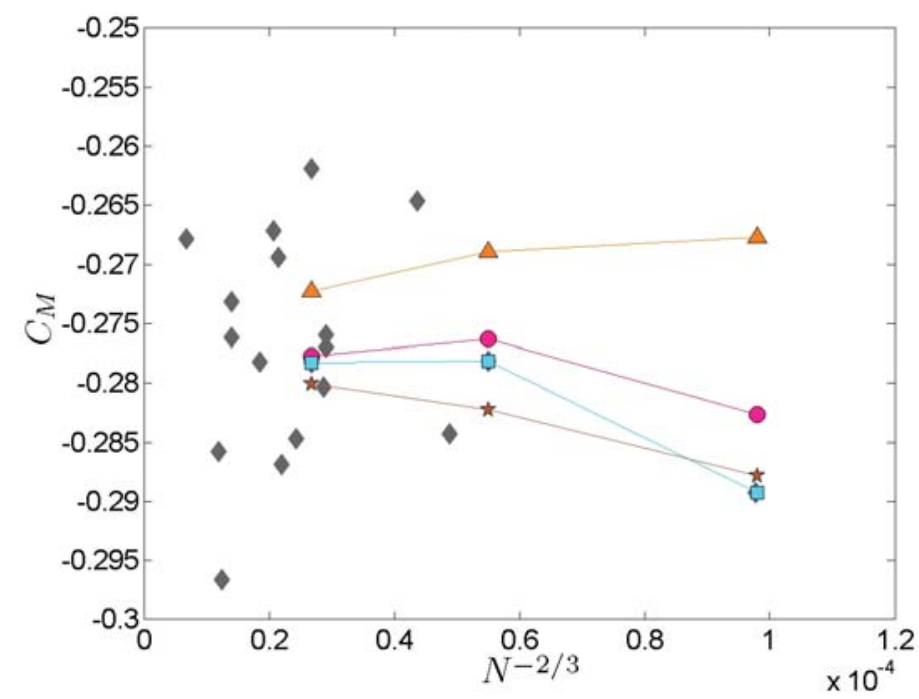

Figure 4. Pitching Moment Coefficients from Static Aeroelastic Solutions.

their own software to generate cutting planes at seven span stations to extract pressure coefficient as a function of a local chord length at each time step. These pressure time histories were then sent to one person to generate the FRFs of pressures due to displacement. Figure 5(b)shows the resulting FRF magnitude for the upper wing surface at 59\% span station (station 4) for the coarse, medium, and fine grids for all flow solvers. The plot shown is zoomed in on the shock location.

The common grid study results show that tight control over the grid, turbulence models and postprocessing produce a reduction in variation among the results, however, no significant reduction in difference relative to the experiment was achieved.

\section{Validation aspect \#2: Postprocessing of computational results}

Postprocessing introduces significant variation into the computational results. One outcome of the closely tracked comparisons from the common grid study, was that a "same-software and the same-person" post processing is recommended at the earliest possible stage. It is recommended that common processing begin from the pressure time histories of the entire wing surfaces and not from the pressure time histories of already extracted and perhaps splined data by each researcher. The influence of several aspects of the postprocessing are discussed below.

In the common grid study, a same-software and same-person post processing method was enforced. Figure 6 presents an example comparing results for different postprocessing procedures that were designed to be identical. The figure shows, in orange color, the FRFs produced from a linearly interpolated point distribution. The blue color line represents the FRFs calculated by one of the AePW participants in preparation for that workshop. The results show some differences in peak values. The pink line was derived from the blue line after additional spline interpolation to extract the $C_{p}$ values at the experimental pressure tab locations.

SPATIAL SLICING EFFECTS The following problem was encountered and solved during postprocessing of the results from the common grid study previously described. The first attempt at computing FRFs from the time histories at each span station obtained from each flow solver produced small spikes on both upper and lower surfaces. An example is presented in figure 7(a) at 32\% span (Station 2). This span station is used as an example only; these spikes were present at other stations too. The figure presents a zoomed in region, shown on the insert by the black circle. Note, that in these figures the $\mathrm{x}$-axis has not been normalized by the chord. The cause of these spikes was traced back to the point distribution obtained from the cutting planes at each station, a common method of data extraction for CFD results. Due to the orientation of the quadrilateral elements on the surface with respect to the cutting plane, every third or fourth point in the extracted plane is very close to another point. Figure 7(b) shows the fine grid with a cutting plane in red color on the lower wing surface. Six locations marked in green show examples where the cutting plane's grid points 


\begin{tabular}{|c|c|c|c|}
\hline \multicolumn{2}{|c|}{$\begin{array}{l}\text { Experiment } \\
-O-\text { Statistical Mode } \\
--\Delta-\cdot \text { Maximum } \\
--\nabla-\cdot \text { Minimum } \\
\end{array}$} & \multicolumn{2}{|c|}{ AePW Computations } \\
\hline \multicolumn{4}{|c|}{ Post-Workshop Computations } \\
\hline & \multicolumn{3}{|c|}{ Grid Resolution } \\
\hline Software & Coarse & Medium & Fine \\
\hline FUN3D & -0 & 0 & -0 \\
\hline TAU & -- & $\square$ & $\square$ \\
\hline Edge & $\boldsymbol{\Delta}-$ & $\Delta$ & $\Delta$ \\
\hline Fluent & $\star$ & - & \# \\
\hline
\end{tabular}

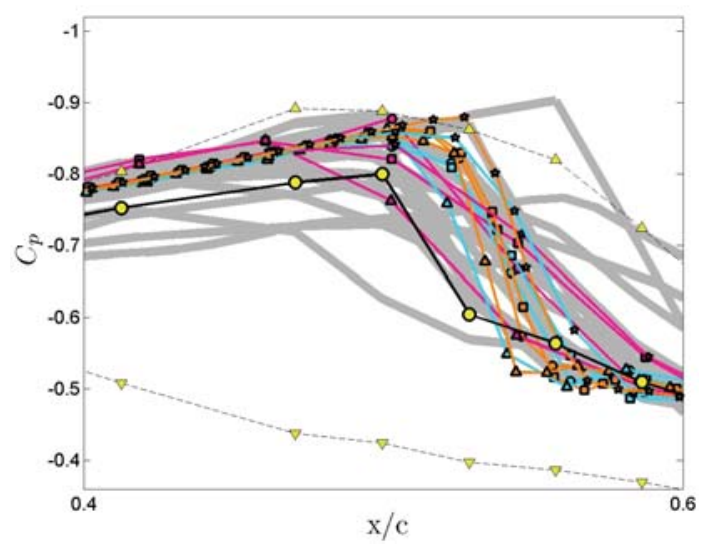

(a) Unforced system; $C_{p}$ distribution

\begin{tabular}{|c|c|c|c|}
\hline$-O-E x$ & ment = & AePW Co & utations \\
\hline & t-Worksh & omputatic & \\
\hline & & d Resoluti & \\
\hline Software & Coarse & Medium & Fine \\
\hline FUN3D & -1 & 0 & 0 \\
\hline TAU & $\rightarrow-$ & $\square$ & $\square$ \\
\hline Edge & $\boldsymbol{\Delta}$ & $-\Delta$ & $-\Delta$ \\
\hline
\end{tabular}

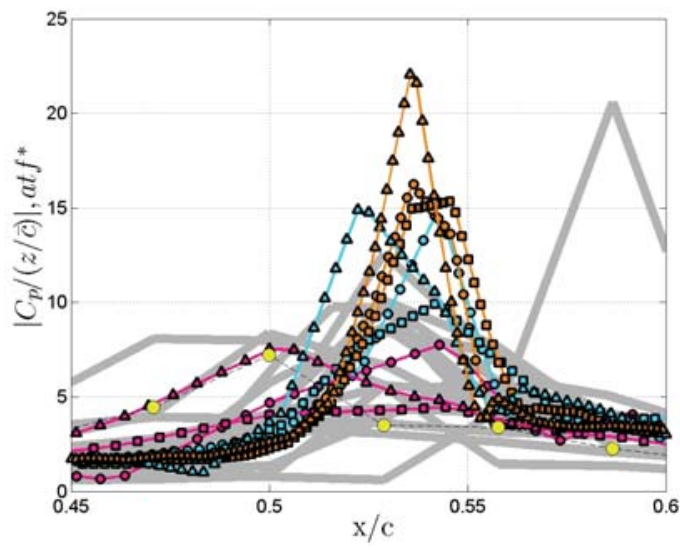

(b) Forced oscillation;FRF Magnitude

Figure 5. HIRENASD common grid aeroelastic solutions; $C_{p}$ for coarse/medium/fine grids, $59 \%$ span station (Station 4 ) shock region, Upper surface.

are very close to each other. These six locations are repeated in figure 7 together with the spikes in FRFs. To mitigate this issue, the cutting plane's grid distribution and its corresponding pressure coefficient values were re-distributed using linear interpolation. The linear interpolation method was applied consistently to the results from all three flow solvers. $^{48}$

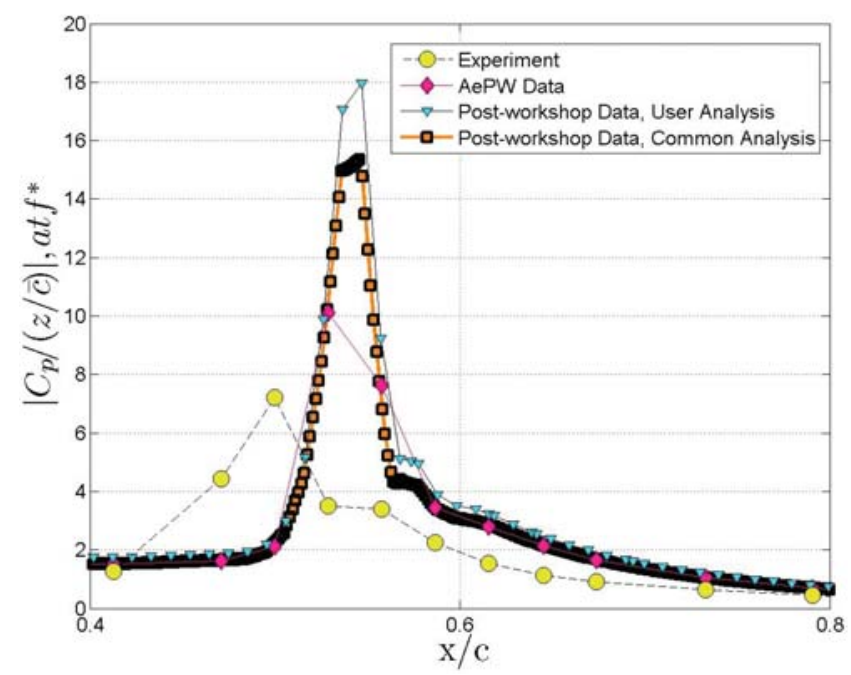

Figure 6. AePW and post AePW FRFs processing, 59\% span station (Station 4), upper surface.

TIME DOMAIN PARAMETERS Successful postprocessing requires attention to both the time spacing between data samples and the time length of the data record. These two time domain requirements can not be satisfied post-solution and must be considered up front in performing the calculations.

The Nyquist frequency defines the maximum frequency of the flow phenomena that can be identified using Fourier analysis. It is determined by the time step size, $\Delta t$, measured in seconds, as shown in equation (1). In order to discern 


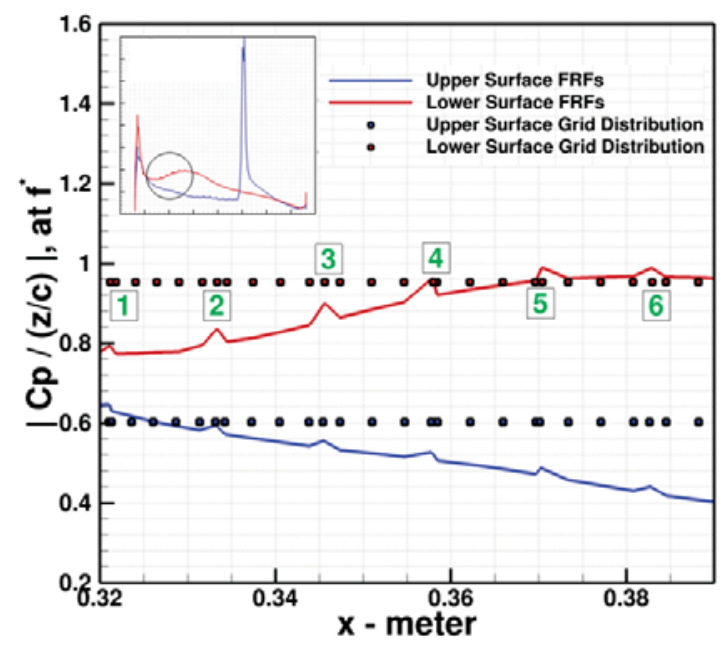

(a) Magnitude of FRFs.

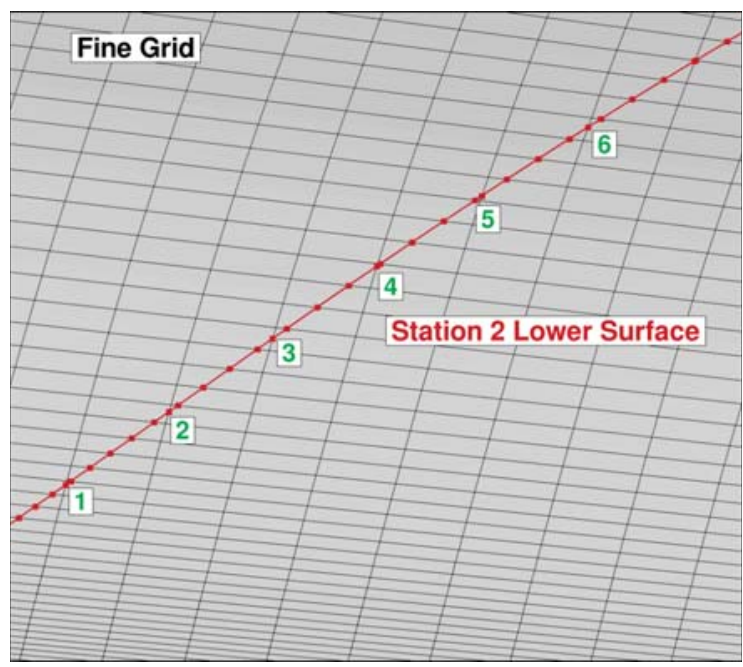

(b) Grid Distribution vs. Cutting Plane.

Figure 7. Grid Distribution and FRFs spikes; HIRENASD, 32\% span station (Station 2).

content at a frequency, the Nyquist requirement is that each cycle must be represented by 2 or more time points. Guidelines for data processing of experimental information advise the data analyst to sample the data at a rate 2 to 5 times faster than the Nyquist criteria would demand, relative to the highest frequency of concern for their application (i.e., desired bandwidth). The guideline of a factor of 5 has also been recommended by CFD data analysts. ${ }^{50}$ Satisfying the requirement that the Fourier analysis can properly resolve the information, however, does not guarantee that the time step size chosen for the solution will be sufficient to model the flow phenomena of interest or produce proper solution convergence. The Nyquist criterion emphasizes that sampling the computational results at well-spaced time points rather than at all global time steps (decimation) is not a recommended practice until the issue of frequency range requirement for an analysis is well understood.

$$
f_{\text {Nyquist }}(H z)=\frac{1(\text { cycle })}{2 \Delta t(\sec )}
$$

The Fourier frequency resolution defines the lowest non-zero frequency that can be resolved using Fourier analysis and determines the spacing of the frequencies available for defining frequency response characteristics. Closely spaced frequencies enable better definition of the peak value, peak frequency and damping characteristics. The Fourier frequency resolution is determined by the time record length on which Fourier analysis is performed, $T_{\text {ensemble }}$, as shown in equation (2). Often, data records are not analyzed as a single record but are broken into overlapping segments for computing the Fourier series coefficients. The FRFs are computed using power spectral density and cross spectral density functions that are average values obtained for all of the ensembles or data segments. ${ }^{58}$ Segmenting the data reduces the achievable frequency resolution but improves the confidence in the results. ${ }^{59}$ The frequency resolution values given in table 2 correspond to the finest resolution achievable for a given data set- the case that uses the entire time record as a single analysis block.

$$
\Delta f(H z)=\frac{1(\text { cycle })}{T_{\text {ensemble }}(\mathrm{sec})}
$$

Tables summarizing the computational solution parameters for each of the submitted analyses have been previously published. ${ }^{35}$ Table 2 shows the parameters for the BSCW computations at $1 \mathrm{~Hz}$ as an example. The table shows the number of time steps per cycle, the number of cycles computed and the number of subiterations that were performed for each global time step. The table lists the physical time length of the computational data records, the maximum Nyquist frequency based on sample rate and the minimum Fourier frequency spacing based on the time record length.

A final point regarding unifying the postprocessing: a common process does not ensure accuracy or reliability of the solutions. Correct application and understanding the underlying assumptions of the postprocessing methods being used is essential as well as understanding the CFD assumptions and processes. 
Table 2. Time Accuracy Computational Parameters, BSCW, $1 \mathrm{~Hz}$ forced oscillations

\begin{tabular}{cccc|ccc} 
Grid & $\begin{array}{c}\text { \# of steps } \\
\text { per }\end{array}$ & $\begin{array}{c}\text { \# of } \\
\text { cycles } \\
\text { computed }\end{array}$ & $\begin{array}{c}\text { \# of } \\
\text { subiters } \\
\text { per global } \\
\text { time step }\end{array}$ & $\begin{array}{c}\text { Time } \\
\text { record } \\
\text { length } \\
(\text { sec })\end{array}$ & $\begin{array}{c}\text { Nyquist } \\
\text { frequency } \\
(\mathrm{Hz})\end{array}$ & $\begin{array}{c}\text { Fourier } \\
\text { frequency } \\
\text { resolution } \\
(\mathrm{Hz})\end{array}$ \\
\hline \hline M & 128 & 5 & -1 & 5 & 64 & 0.20 \\
C & 128 & 8 & 25 & 8 & 64 & 0.13 \\
M & {$[128,256,1024]$} & {$[8,4,2]$} & 25 & {$[8,4,2]$} & {$[64,128,512]$} & {$[0.13,0.25,0.50]$} \\
F & {$[128,1024]$} & {$[8,2]$} & 25 & {$[8,2]$} & {$[64,512]$} & {$[0.13,0.50]$} \\
M & {$[200,400,800]$} & {$[9,9,3]$} & {$[6,4,4]$} & {$[9,9,3]$} & {$[100,200,400]$} & {$[0.11,0.11,0.33]$} \\
C & {$[180,360,720,720]$} & 4 & {$[20,20,20,50]$} & 4 & {$[90,180,360,360]$} & 0.25 \\
M & 720 & 4 & 50 & 4 & 360 & 0.25 \\
C & 200 & 10 & 20 & 10 & 100 & 0.10 \\
M & 200 & 10 & 20 & 10 & 100 & 0.10 \\
F & 200 & 10 & 20 & 10 & 100 & 0.10 \\
$*$
\end{tabular}

* Coarse (C); Medium (M); Fine (F)

\section{Validation aspect \#3: Aeroelastic considerations}

The degree of coupling between the fluid and the structure is dependent on many variables: flow field force distribution (pressure distribution); flow field strength (dynamic pressure); geometric presentation of the structure to the flow field (deformation distribution); and magnitude of the deformation. For the aerodynamic problem to be completely uncoupled from the structural considerations, the structure must be perfectly rigid, without any elastic deformation. This is an idealization, as all real structures are flexible under loading. Weakly coupled systems are designated as those systems that have small influences of the structural deformation on the aerodynamics, or small influences of the aerodynamics on the structure. Most aerodynamic studies assume that the model is completely rigid and neglect all influence of the structural deformation. For the AePW, the RSW or the BSCW were treated as rigid configurations.

The HIRENASD was analyzed as a flexible system, incorporated into both the unforced (static aeroelastic) results and the forced oscillation results. The majority of these aeroelastic computations were performed using a modal representation of the structure. The modes were obtained from a linear structural dynamic analysis of a finite element model and interpolated to an aerodynamic surface mesh. The mode-based aeroelastic solutions were performed in three steps. First, the steady CFD solution was obtained for the rigid body. Next, a static aeroelastic solution was obtained by continuing the CFD analysis in a time accurate mode with a structural modal solver, allowing the structure to deform. Finally, for the dynamic response, a user-specified modal motion was used. In this study, for harmonic perturbation, the modal displacement for only the second mode was applied by almost all analysis teams.

There were several exceptions to this process: one HIRENASD analysis team performed direct-coupled simulations using the full finite element model; another HIRENASD analysis team performed both a modal solution and directly-coupled solutions with a simplified structural dynamic model; and one team investigated the influence of incorporating all modes into the solution and the influence of changes to the structural dynamic model.

A finite element model (FEM) of the HIRENASD configuration used for AePW was a modified version of a model provided by RWTH Aachen University. ${ }^{60}$ The modifications include adding the mass of the instrumentation, better simulating the bolt connections and incorporating mount system hardware. The result is a very detailed model, containing over 200,000 uniform solid hexagonal elements in the wing alone. Specific details of the FEM are described by Wieseman. ${ }^{61}$ The modified FEM was validated by comparing modal frequencies, modal assurance criteria, comparing leading edge, trailing edge and twist of the wing with data obtained from experiment. There was a significant change in the frequency of the second bending mode with minor impact on the other modal frequencies. Also, the second bending mode node line shifted inboard, bringing it closer to agreement with experimental data. The largest changes in modal character were produced by the explicit modeling of the mount system components.

Normal modes analysis was performed on the modified FEM and the first 30 modes extracted for use in the aeroelastic computations. The effort documented by Wieseman ${ }^{61}$ increased confidence in the structural dynamic rep- 
Table 3. Solution processes for AePW configurations

\begin{tabular}{|c|c|c|c|c|c|c|}
\hline \multirow[b]{2}{*}{ Configuration } & \multicolumn{3}{|c|}{ Unforced System } & \multicolumn{3}{|c|}{$\begin{array}{c}\text { Forced Oscillation System, } \\
\text { Time-accurate solutions }\end{array}$} \\
\hline & $\begin{array}{l}\text { Steady, } \\
\text { Rigid }\end{array}$ & $\begin{array}{c}\text { Steady, } \\
\text { Static } \\
\text { aeroelastic }\end{array}$ & $\begin{array}{c}\text { Time- } \\
\text { accurate, } \\
\text { Rigid }\end{array}$ & $\begin{array}{l}\text { Unsteady } \\
\text { aerodynamic }\end{array}$ & $\begin{array}{c}\text { Unsteady } \\
\text { aerodynamics on } \\
\text { deformed static } \\
\text { aeroelastic mesh }\end{array}$ & $\begin{array}{c}\text { Unsteady } \\
\text { aeroelastic } \\
\text { response }\end{array}$ \\
\hline RSW & $\checkmark$ & & & $\checkmark$ & & \\
\hline BSCW & $\checkmark$ & & $\checkmark$ & $\checkmark$ & & \\
\hline HIRENASD & & $\checkmark$ & & $\checkmark^{\dagger}$ & $\checkmark$ & $\checkmark^{*}$ \\
\hline
\end{tabular}

resentation and demonstrated the insensitivity of the results to small modifications or errors in the structural modeling. This was crucial to the Aeroelastic Prediction Workshop in that differences of the CFD results from different analysts as compared to experiment could then be clearly discussed in terms of aerodynamic differences and not structural modeling.

As mentioned previously, one team desired to perform a direct coupling between the aerodynamic and structural models. Because the FEM external surface definition did not originally match the aerodynamic outer mold line (OML) an additional modification of the FEM was required. The FEM grid points were projected to the aerodynamic OML. The most troublesome region on the HIRENASD geometry was the trailing edge of the wing tip. The level of detail that is required is illustrated in figure 8. This is a change to the usual process that has historically been employed in aeroelastic analysis. It represents an additional and non-trivial requirement to be placed on the structural modeling effort.

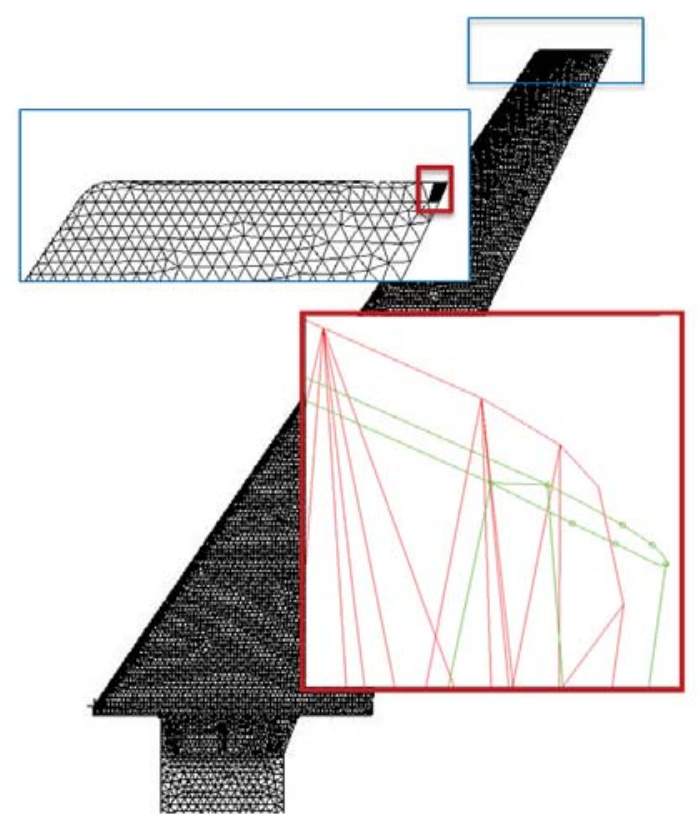

Figure 8. Mapping the outer surface of the finite element model to the IGES file for directly coupled aeroelastic analyses; red line shows the structural finite element model prior to OML mapping; green lines show the aerodynamic geometry. Note that only the wing portion of the FEM is shown here.

Post-workshop, an analysis team conducted aeroelastic analyses using the finite elelement model before and after the modifications described above, using a common grid and identical solution parameters. ${ }^{29}$ The results showed indiscernable differences in the static pressure distributions using 30 flexible modes from each of the two FEMs. For the HIRENASD configuration at this test condition, the dynamics are dominated by the shock oscillations on the upper surface. At the inboard-most span station on the lower surface, there is also an oscillating shock. This is mentioned at this stage because it is in this region (the inboard lower surface) where the largest magnitude difference in aeroelastic 
response was produced by the changes in the finite element model. The change in the magnitude of the response is on the order of $10 \%$ at the peak magnitude location. This is illustrated in figure 9 , by direct comparison of the two magnitude plots. The differences on the upper surface in the region of the dynamic shock motion are considerably less. Analysis of pressure coefficient data for both upper and lower surface at seven span stations on the wing show larger percent differences for the leading and trailing edge dynamic responses than for the shock dynamics. These larger percentage differences are thus inflated by the low values of the responses in these regions.

The aeroelastic analysis results show that for the weakly coupled HIRENASD, oscillated near the modal frequency, the results were insignificantly sensitive to the mode shape, and demonstrated no sensitivity to the modal frequency. Neither the static aeroelastic results nor the second bending forced excitation results changed significantly by changing the FEM. This is not anticipated to be a finding that is extendable to more highly coupled aeroelastic systems. In considering whether or not the results will likely be sensitive to the modal frequency, one indicator in an experimental data set is a large shift in the frequency from the air-off condition to the test condition.

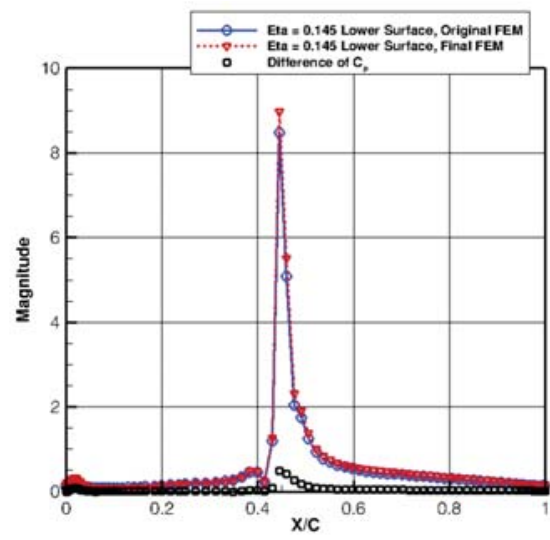

Figure 9. Magnitude of unsteady pressures and the difference of pressure coefficients, inboard span station (14.5\% span), lower surface

\section{Validation aspect \#4: Representing unsteady information}

Formulating a workshop includes debating what type of data to request from the computational teams and how to process and present the experimental comparison data. The following sections discuss some of the issues that arose regarding both the unforced and forced oscillation data sets.

UNSTEADY CONTENT OF UNFORCED SYSTEM DATA SETS The experience of the AePW team is that unforced system data does contain dynamics including large changes in pressure coefficients on the upper surface as the shock moves. This issue was illustrated by each configuration in separate publications. ${ }^{15,19,37}$ Unforced system data sets have sometimes been termed "steady" or "static" or "stationary," but bounds on the $C_{p}$ data are quite large, particularly near the upper surface shock. The variability seemed excessive at first glance when compared with data sets used for other CFD comparison workshops. Recent discussions among those involved in the HIRENASD testing and data reduction continue to explore improved methods of characterizing the data for both the unforced and forced oscillation systems. Some of these discussions and results are presented below. There are two related issues: representing the expected distribution (usually characterized by a profile of mean values along the chord), and representing the variation about the expected value profile.

Originally, the experimental unforced system information presented for comparison was described using mean values. The presence of shock motion in a presumed static data set has been documented to have the following effects on the resulting mean pressure distribution: smearing the shock over several chord stations, reducing the magnitude of the shock, and canting the shock towards the leading edge.

A mean value only adequately represents a time history data set if that time history has a symmetric distribution. Often, use of the mean value is accompanied by the assumption that the data is Gaussian distributed. In the case of pure oscillations, the data distribution is symmetric so the mean is a reasonable quantity to represent the information. In the case of an oscillatory shock, however, the pressure measured by a sensor or calculated at a grid point in the spatial range of the oscillation can have a highly skewed distribution- not symmetric and poorly represented by statistical quantities associated with a Gaussian distribution. 
UnSTEADY SHOCK MOTION Transonic conditions generate shocks; in the AePW data sets all of the shocks were oscillatory to some extent. The pressure time histories in the regions of the oscillating shocks appear as amplitudebounded nonlinear traces for sensors that lie within the range of the shock motion. This bounded characteristic is illustrated by the BSCW at the AePW test conditions for the unforced and $10 \mathrm{~Hz}$ forced oscillation cases in figures 10(a) and 10(b). The vertical axis in each of these figures was inverted to correspond to the usual plotting convention of the pressure coefficient. The lower limits on the pressure plots correspond to the pressure levels that exist at the foot of the shock; the upper limits on the plots correspond to the pressure levels ahead of the shock. Statistical mode values, calculated from each of the pressure transducer time histories, also indicate the shock location. Chord-wise distributions of the pressure coefficient are shown in figure 11, where the abrupt changes in pressure near $x / c=0.5$ for both the unforced system and the forced oscillation system indicate the location of the shock.

These characteristics also occur for attached flow cases. A test condition (Mach 0.7, $\alpha=5^{\circ}$ ) that was not part of the AePW test matrix is used to illustrate the behavior of the attached flow cases in figures 10 (c) and 10(d). The pressure coefficient distributions for these cases are also shown using statistical mode values in figure 11.

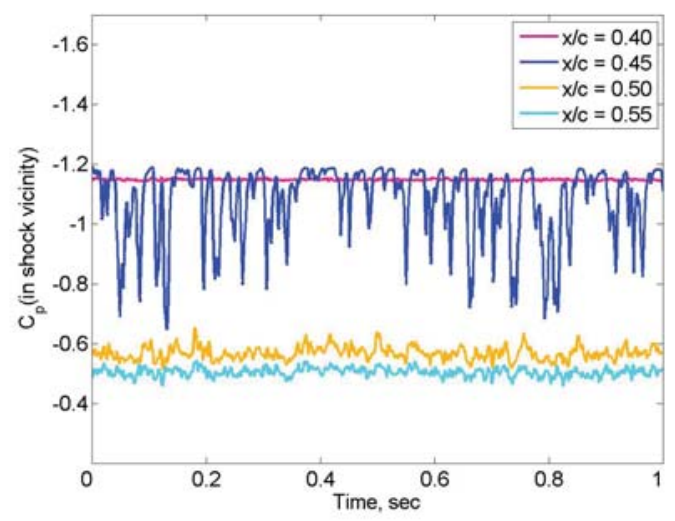

(a) Separated flow, unforced case (Mach $0.85 \alpha=5^{\circ}$ )

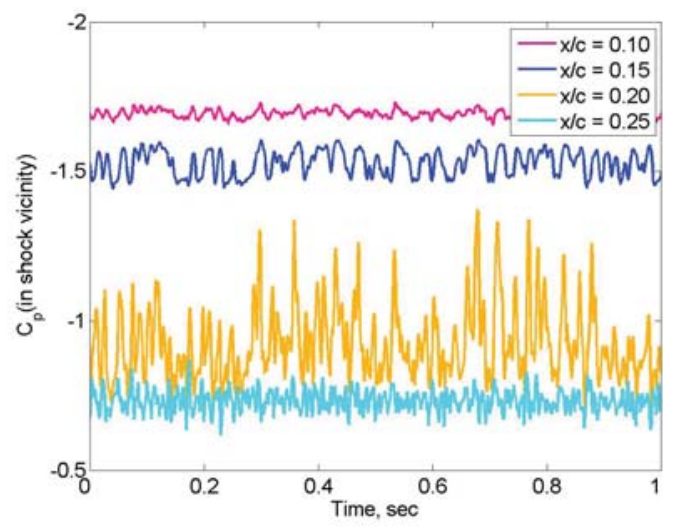

(c) Attached flow, unforced case (Mach 0.7, $\alpha=5^{\circ}$ )

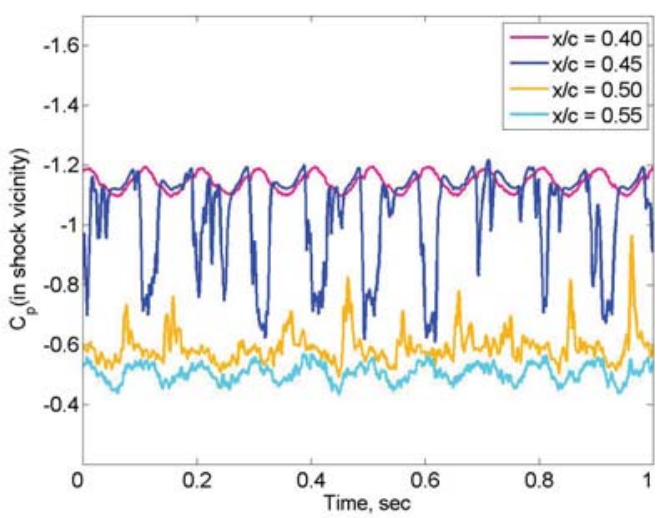

(b) Separated flow, $10 \mathrm{~Hz}$ forced oscillation (Mach $0.85, \alpha=5^{\circ}$ )

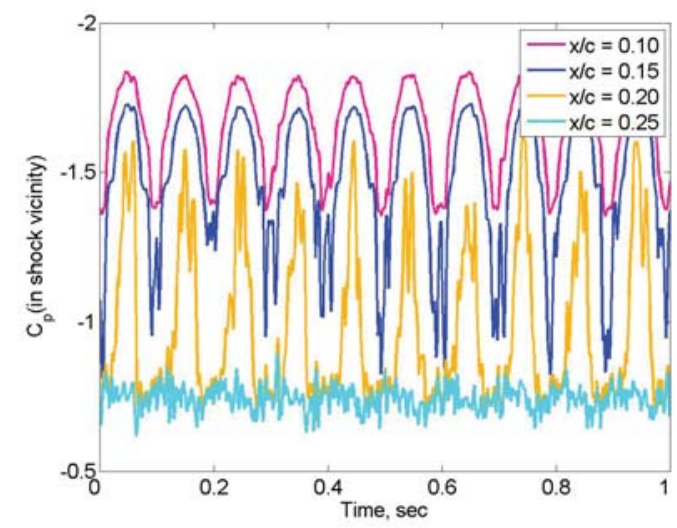

(d) Attached flow, $10 \mathrm{~Hz}$ forced oscillation (Mach 0.7, $\alpha=5^{\circ}$ )

Figure 10. Time histories of pressure coefficients in the vicinity of the shock, BSCW, Upper surface, $\eta=0.59$.

The bounded time histories associated with the traversing shock give rise to skewed histograms rather than symmetric bell-shaped histograms associated with Gaussian distributions. Asymmetry of histograms is statistically characterized by the skewness. ${ }^{62}$ When data is spread out more to the right of the mean value than to the left, the skewness has a positive value. That is, a data set with a left-skewed peak and an extended right-side tail will have a positive value of skewness. Unforced system data from the HIRENASD configuration is shown using histograms in figure 12 and skewness in figure 13.

A left-skewed peak on the histogram indicates that the sensor is ahead of the shock more often than not; its usual position in the pressure distribution is on the supersonic plateau indicated by large negative values. Points in time when the sensor has its customary value generate the principal peak in the histogram. Examples of this characterization are 


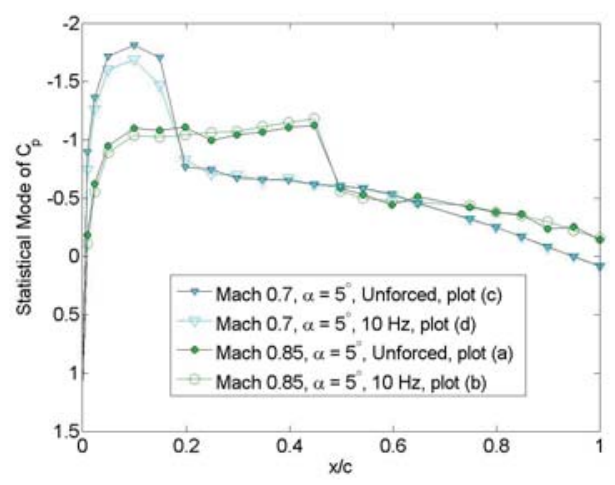

Figure 11. Statistical mode distribution, BSCW, Upper surface, $\eta=0.59$.

shown for sensors 5 and $6,(x / c=0.41$ and 0.47$)$, in figures 12 (a) and 12(b). Occasionally, the shock oscillates forward and the sensor is then aft of the shock in the subsonic flow area at the foot of the shock, at a smaller negative or positive pressure coefficient value. These subsonic values form the right-side tail of the histogram. For points that are primarily aft of the shock, the histogram has a right-skewed peak and an extended left-side tail.

The histogram of data at $\mathrm{x} / \mathrm{c}=0.5$, figure 12 (c), has a bi-modal character. For systems that are sinusoidally forced, the bi-modal shape is a common characteristic of the histogram; an arcsine histogram is the expected distribution corresponding to a sinusoidal time history. The data represented in figure 12, however, is an unforced data set. Here, the bi-modal character shows that the sensor is fully crossed by the shock. This can indicate either that the shock is traversing a large chord-wise range or that the shock is very sharp. It is unclear from just the histogram which case reflects the true situation.

The skewess plot helps to define the "most likely" location of the center of the shock, although in this case the bimodal character of the histogram at the midchord sensor is a direct sign that the center of the shock is very near this sensor. Interpolating the skewness information, the maximum likelihood location for the shock center is just aft of the midchord sensor, at $\mathrm{x} / \mathrm{c}=0.505$.

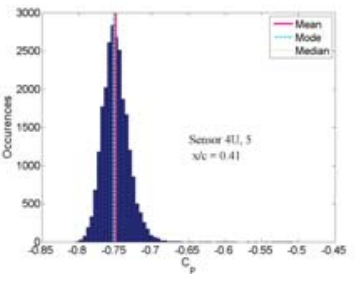

(a) Sensor $5, \mathrm{x} / \mathrm{c}=0.41$; Sensor is forward of the shock for nearly all time points.

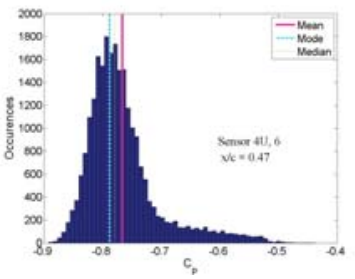

(b) Sensor 6, $\mathrm{x} / \mathrm{c}=0.47$; Sensor is forward of shock for most time points.

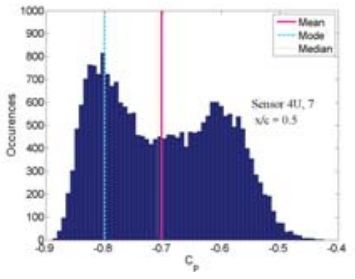

(c) Sensor 7, $\mathrm{x} / \mathrm{c}=0.50$; Sensor is crossed by the shock.

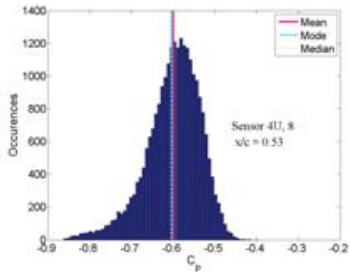

(d) Sensor $8, \mathrm{x} / \mathrm{c}=0.53$; Sensor is aft of the shock for most time points.

Figure 12. Histograms of unforced system responses; HIRENASD, Mach 0.8, Reynolds number $\left(\operatorname{Re}_{c}\right) 7$ million, $\alpha=1.5^{\circ} ; \mathbf{5 9 \%}$ span $($ Station 4$)$, Upper surface

The consequence of using mean values to represent nonsymmetric pressure coefficient data is that the distribution will not represent the most likely distribution. Three types of mis-representation have been observed in the AePW data sets, generated by using mean values to represent the pressure distribution: 1)The pressure associated with the moving shock gets smeared over several chord stations when the mean value is used; 2) Because the mean will also represent an averaged magnitude, the overall magnitude of the pressure change across the shock is reduced relative to the actual difference between the end of the supersonic plateau and the foot of the shock; and 3) Because the pressure magnitude is higher ahead of the shock than behind the shock, averaging the pressures together as the shock moves across a sensor or a grid point resultd in the pressure distribution change across the shock slanting towards the leading edge, rather than being more vertical.

STATISTICAL MODE, BOUNDS AND SMEAR PLOTS FOR OSCILLATORY SOLUTION TIME HISTORIES The current AePW data processing standard is to calculate the statistical mode as the first statistical moment in the vicinity of the shock, rather than the mean. Alternatively, Boucke ${ }^{63}$ employs a kernel density estimate method to represent the 


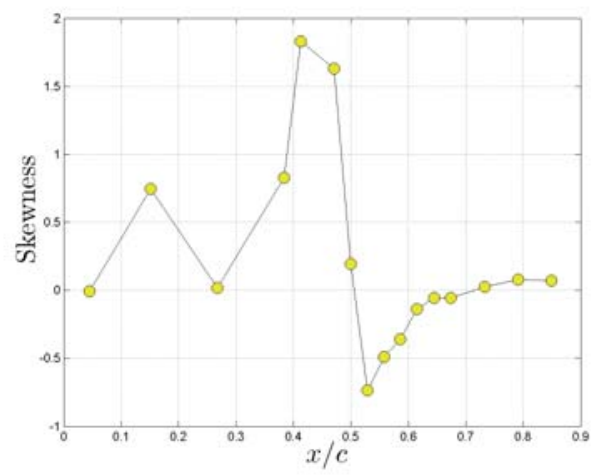

Figure 13. Skewness, HIRENASD 59\% span station, upper surface.

expected value of the system. The influence of these alternate characterizations of the first statistical moment of the data is to sharpen the shock (make it more vertical) and show it as stronger (larger difference between pressure coefficient ahead of and behind the shock) in comparison to the mean value representation. These alternate representations, though, do not capture the range of motion of the shock or the variation about the expected value profile.

This variation has been represented in several ways during the AePW data analysis process. Initially, when Gaussian distribution statistics were used to represent the data, ${ }^{46}$ bounds of two standard deviations relative to the mean were displayed. In the year since the workshop, results have principally displayed bounds showing the data maxima and minima for each chord location. In some plots, the $99.5 \%$ and $0.5 \%$ capture bounds were used as bounds rather than the strict maxima and minima. ${ }^{19,37}$ An example using HIRENASD data is shown in figure 14. Experimental data time history examples at 59\% span (station 4) are shown as a function of chord location in figures 14(a) and 14(b) and as a function of time in figure 14(c). In figures 14(a) and 14(b), each of the grey lines represents a different point in time. Here, every $100^{\text {th }}$ time point is plotted. The mean, mode, maximum and minimum profiles, computed using the entire time record are also shown. As the capture bounds are constricted to eliminate the more outlying values, the plotted bounds squeeze in and eliminate white space. The three raspberry-colored lines in figures 14(a) and 14(b) are snapshots at specific, arbitrarily chosen, times. Subsets of the time histories associated with sensors at three chord locations are plotted in figure 14(c). In this figure, the time points corresponding to the snapshots of the previous figures are identified by the raspberry-colored circles. These plots show that the bounds included in figures of unforced system data indicate the dynamic content of the signals.

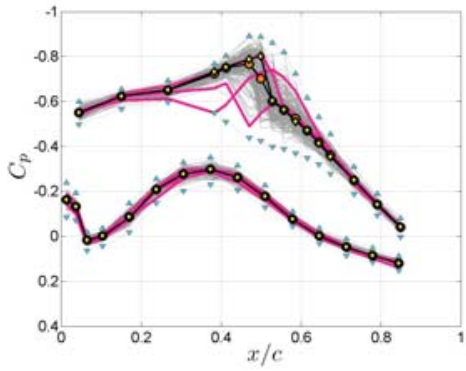

(a) Time histories and statistics

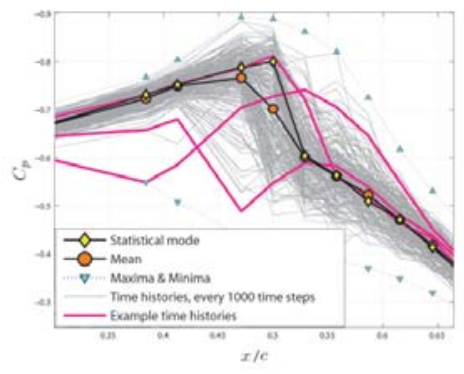

(b) Focus on the upper surface shock region

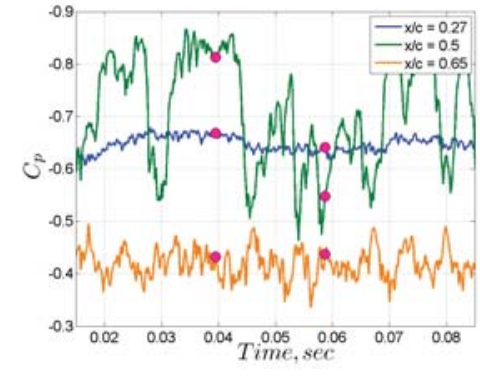

(c) Time histories of selected sensors

Figure 14. HIRENASD, experimental data of the unforced system, 59\% span (Station 4)

WHY OUR DATA LOOKS DIFFERENT The data sets for the AePW present a more accurate picture of the dynamic content that is present than other published data sets for benchmarking workshops due to the instrumentation, data acquisition systems and data processing methods. Example comparison data sets for the HIRENASD and the Common Research Model (CRM), associated with the Drag Prediction Workshops, ${ }^{6,7}$ are shown in figure 15 for a $0.4^{\circ}$ change in angle of attack. For the AePW data sets, the instrumentation and data acquisition systems were capable of measuring and recording data minimally at $1000 \mathrm{~Hz}$; the time history records were saved and used to generate the response bounds. Experiments that focus on static aerodynamic quantities typically emphasize time-averaged quantities. For 
the CRM data set, generally only the mean values calculated from pressure sensors with $5 \mathrm{~Hz}$ Nyquist frequency are available. Figure 15(b) shows the mean values with yellow triangles and the time-varying data for the low-bandwidth sensors with the magenta circles. The CRM contains only one high-frequency pressure sensor, located near $\mathrm{x} / \mathrm{c}=$ 0.5 near CRM span station 7 (73\% span station). This data was recorded by a high frequency data acquisition system during designated test points. This higher frequency data is shown by the blue dots and indicates that the low-frequency nature of the other measurements filters out a substantial portion of the pressure response; the range of these values shows that the CRM pressure fluctuations exceed those documented in the HIRENASD data. Bounds on the response at other locations can not reasonably be estimated using the bounds provided at this chord location, however.

Although models intended for steady aerodynamic tests can be expected to contain some of the dynamics that are encountered in aeroelastic testing, there are additional possible sources of increased dynamic response levels that may be present in aeroelastic systems. These sources include: 1) structural dynamic modes designed to be in the range of the measurements; 2) freeplay within the model actuation and mounting systems; and 3) excitation signals which have not been reduced to machine zero during the unforced system testing.

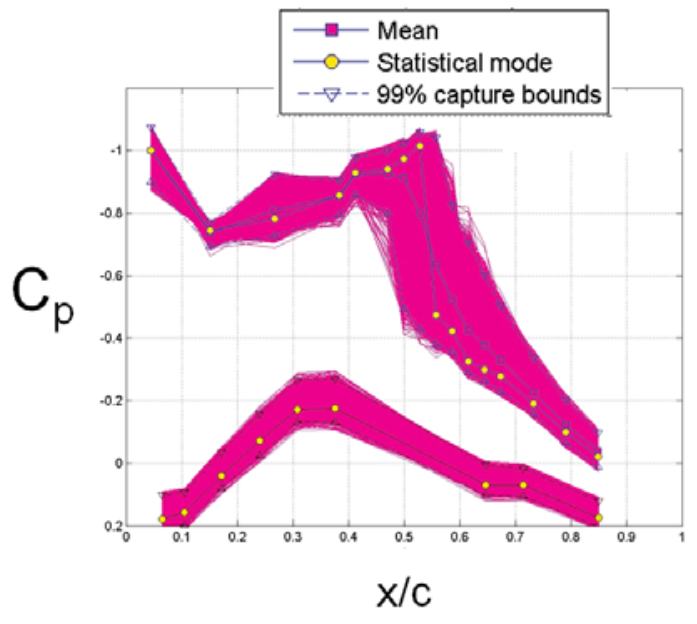

(a) HIRENASD, 59\% span (Station 4)

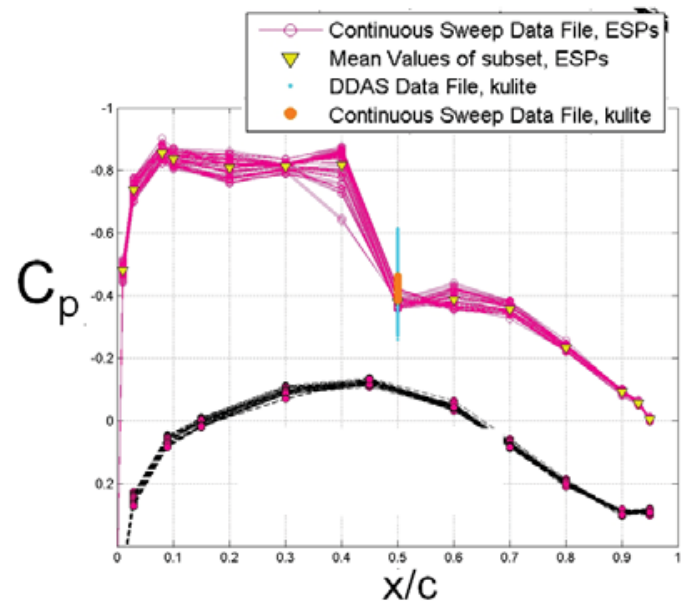

(b) Common Research Model, 73\% span (Station 7)

Figure 15. Comparison of HIRENASD and CRM dynamic content, varying angle of attack from $2.8^{\circ}$ to $3.2^{\circ}$

INFLUENCE OF FORCED OSCILLATION ON SMEAR PLOT Smear plots from the unforced system and the forced oscillation case are compared for example data sets from the BSCW in figure 16. The range of the pressure coefficient for the forced system is always greater than that of the unforced system. In the figure, this is shown for the upper surface by the forced oscillation data (grey lines) always banding the unforced system data (black lines). Similarly, for the lower surface data sets. Forward of the upper surface shock, the unforced system response range is narrow relative to the forced oscillation result. However, aft of the shock, the forced oscillation response range barely exceeds that of the unforced system. This emphasizes the flowfield is separated aft of the shock and that most of the dynamic response in this region is associated with the aerodynamic separation physics rather than with the forcing function of the structure.

INTEGRATED OR ROLLED-UP QUANTITIES The lift, drag and pitching moment integrate information over the entire wing. A positive aspect in comparing these integrated quantities is that they can provide good summary comparisons. The negative aspect is that the integration process masks local dynamics. Integrated coefficients can also be calculated for specified subsections of the wing- that is, integrating only in the chordwise direction. For aeroelastic analysis it has been suggested that these sectional coefficients be computed as primary comparison quantities. This has not been done to date, although the suggestion is viewed as a favorable compromise between comparison of detailed pressure coefficient distributions and fully integrated coefficients.

\section{Concluding remarks}

The AePW effort was successful in addressing the primary questions posed regarding benchmarking unsteady aerodynamic modeling. Areas of successes and shortcomings were identified both in computations and experiments. 


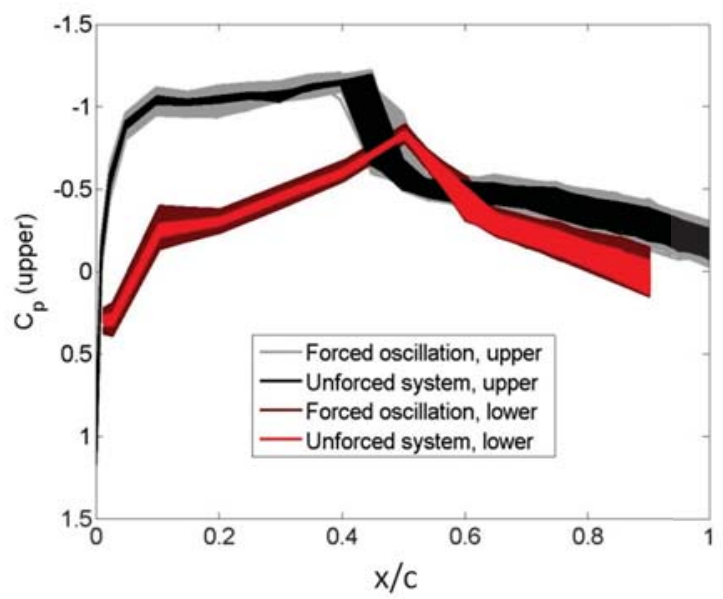

Figure 16. Comparison of smear plots for unforced and forced oscillation data sets; BSCW, $10 \mathrm{~Hz}$

The discussions in this paper focused on several aspects of the workshop related to validation of unsteady computations and experiments. The three focus topics related to the computational efforts were reducing variation in the computational modeling choices, reducing differences in the postprocessing and modeling the static aeroelastic effects. The focus topic regarding the experimental data was how to best represent the information for comparison with the computational results, including the influences of unsteady information in presumed steady data sets.

To improve the quality of the benchmarking and move towards a validation effort, some suggested changes to the workshop process are offered.

- Establish a benchmarking case in the clearly subsonic, clearly attached flow regime

- Eliminate configurations where the experimental data sets are dominated by wall effects

- The scope of the workshop should be focused: a single configuration chosen and a clearly defined and understood goal.

- Establish better guidelines for the computations; make the guidelines available early

- Include solution convergence criteria in the computational guidelines

- Encourage variety in solution fidelity: lower fidelity (panel methods, Euler solutions) and higher fidelity (hybrid RANS-LES, LES) particularly for separated flow cases

- Unify the post processing:

- Have a dedicated postprocessing analyst

- Request time histories of solutions submitted rather than postprocessed data

- Ensure consistency of normalizations, integration areas

- Expand postprocessing methods beyond Fourier techniques

- Choose unsteady solution comparison quantities that capture the critical features shock location, range, strength

- Calculate integrated sectional moment coefficients as comparison quantities

Recommendations for a new experimental validation data set have come from both those involved in the computational and experimental data aspects of the AePW. An ideal data set would contain simultaneous measurements of time-dependent structural deformation, integrated loads, unsteady pressures, skin friction coefficients, and off-body flow fields. There are several differences between the experiments that were conducted and the physics that were modeled. Ideally, these differences would be resolved by either modifying the experiment or by modifying the computations. These differences include presence of wind tunnel walls and splitter plates, metric portions of the mounting system, flow transition from laminar to turbulence, presence of structural dynamics in assumed rigid systems and spatial distribution of information. 


\section{Future directions}

There are many possible and interesting future directions for aeroelastic benchmarking efforts. One of the areas that we may now be in a good position to consider is evaluating methods to model separated and reattaching flow on aeroelastic stability. High fidelity computational methods may provide sufficient accuracy to predict the unsteady separated flow loads, including correct phasing representation. A study regarding the influence of the forcing frequency on the shock motion's contribution to the aeroelastic stability is a recommended intermediate step. Productive future directions would also include working to improve the solutions and quantify the sources of errors in flow regimes where the RANS solutions are considered reasonable. A major gap in the AePW effort is that no one performed linear panel method solutions. Solutions of this type would provide baselines to directly demonstrate the changes or improvements offered by moving from the current state of practice to the current state of the art. The AePW activity has also illustrated the desparate need for a high-quality experimental aeroelastic validation data set that incorporates modern testing and measurement techniques.

\section{Acknowledgments}

The authors gratefully acknowledge the AePW team, particularly the computational teams and the AePW Organizing Committee for their guidance and discussions.

\section{References}

${ }^{1}$ Oberkampf, W. and Trucano, T., "Verification and validation in computational fluid dynamics," Tech. Rep. SAND2002-0529, Sandia National Laboratories, Albuquerque, New Mexico, 2002.

${ }^{2}$ Roy, C. and Oberkampf, W., "A complete framework for verification, validation and uncertainty quantification in scientific computing," Tech. rep., 48th AIAA Aerospace Sciences Meeting, Jan. 4-7, 2010, Orlando, Florida.

${ }^{3}$ Anon., "Guide for the verification and validation of computational fluid dynamics simulations," Tech. rep., American Institute of Aeronautics and Astronautics, AIAA-G-077-1998, Reston, VA, 1998.

${ }^{4}$ Anon., "Assessment of experimental uncertainty with application to wind tunnel testing," Tech. rep., American Institute of Aeronautics and Astronautics, S-071A-1999, Reston, VA, 1999.

${ }^{5}$ Rahaim, C., Oberkampf, W. L., Cosner, R., and Dominik, D., "AIAA committee on standards for computational fluid dynamics- status and plans," AIAA Paper 2003-844, 41st Aerospace Sciences Meeting, Jan. 6-9, 2003, Reno, Nevada.

${ }^{6}$ Levy, D. W. et al., "Summary of Data from the First AIAA CFD Drag Prediction Workshop," AIAA Paper 2002-0841, Jan. 2002.

${ }^{7}$ http://aaac.larc.nasa.gov/tsab/cfdlarc/aiaa-dpw/, August 2010.

${ }^{8}$ Rumsey, C. L. et al., "Summary of the First AIAA CFD High Lift Prediction Workshop," AIAA Paper 2011-0939, Jan. 2011.

${ }^{9}$ http://hiliftpw.larc.nasa.gov/, August 2010.

${ }^{10}$ Ricketts, R. H., Sandford, M., Seidel, D., and Watson, J., "Transonic pressure distributions on a rectangular supercritical wing oscillating in pitch," Journal of Aircraft, Vol. 21, No. 8, 1984.

${ }^{11}$ Ruiz-Calavera, L. et al., "Verification and validation data for computational unsteady aerodynamics," RTO TR-26, Oct. 2000, Report of the Applied Vehicle Technology Panel (AVT) Task Group AVT-010.

${ }^{12}$ Ricketts, R. H., Sandford, M. C., Seidel, D. A., and Watson, J. J., "Transonic Pressure Distributions on a Rectangular Supercritical Wing Oscillating in Pitch," NASA TM 1983-84616, March 1983.

13"http://www . aeronautics.nasa.gov/atp/facilities/tdt/index.html".

${ }^{14}$ Cole, S. R. et al., "Test Activities in the Langley Transonic Dynamics Tunnel and a Summary of Recent Facility Improvements," AIAA Paper 2001-1958, April 2001.

${ }^{15}$ Heeg, J., Chwalowski, P., Wieseman, C., Florance, J., and Schuster, D., "Lessons learned in the selection and development of test cases for the first aeroelastic prediction workshop: Rectangular Supercritical Wing," AIAA-2013-0784, 51st AIAA Aerospace Sciences Meeting, Grapevine, TX, Jan 2013.

${ }^{16}$ Bennett, R., Eckstrom, C., Rivera, J. J., Farmer, M., and Durham, M., "The Benchmark Aeroelastic Models Program- Description and Highlights of Initial Results," NASA Technical Memorandum -104180, Dec. 1991.

${ }^{17}$ Dansberry, B., Durham, M., Bennett, R., Turnock, D., Silva, W., and Rivera, J. J., "Physical properties of the Benchmark models program supercritical wing," NASA Technical Memorandum -4457, 1993.

${ }^{18}$ Piatak, D. and Cleckner, C., "Oscillating Turntable for the measurement of unsteady aerodynamic phenomenon," Journal of Aircraft, Vol 14, No. 1, Jan-Feb 2003.

${ }^{19}$ Heeg, J. and Piatak, D., "Experimental data from the Benchmark SuperCritical Wing wind tunnel test on an oscillating turntable," AIAA2013-1801, 2013, 54th AIAA/ASME/ASCE/AHS/ASC Structures, Structural Dynamics, and Materials Conference, Boston, Massachusetts, Jan. 8-11, 2013.

${ }^{20}$ Ballmann, J., Dafnis, A., Korsch, H., Buxel, C., Reimerdes, H.-G., Brakhage, K.-H., Oliver, H., Braun, C., Baars, A., and Boucke, A., "Experimental Analysis of High Reynolds Number Aero-Structural Dynamics in ETW," AIAA Paper 2008-841, Jan. 2008.

${ }^{21}$ Ballmann, J., Boucke, A., Dickopp, C., and Reimer, L., "Results of Dynamic Experiments in the HiReNASD Project and Analysis of Observed Unsteady Processes,” Tech. Rep. IFASD-2009-103, June 2009.

${ }^{22}$ Dafnis, A., Korsch, H., Buxel, C., and Reimerdes, H.-G., "Dynamic Response of the HiReNASD Elastic Wing Model under Wind-Off and Wind-On Conditions," Tech. Rep. IFASD-2007-073. 
${ }^{23}$ Reimer, L., Braun, C., Chen, B.-H., and Ballmann, J., "Computational Aeroelastic Design and Analysis of the HiReNASD Wind Tunnel Wing Model and Tests," Tech. rep., Stockholm, 2007, International Forum on Aeroelasticity and Structural Dynamics.

${ }^{24}$ Reimer, L., Ballmann, J., and Behr, M., "Computational Analysis of High Reynolds Number Aerostructural Dynamics (HiReNASD) Experiments," Tech. Rep. IFASD-2009-132, Seattle, 2009, International Forum on Aeroelasticity and Structural Dynamics.

${ }^{25}$ Neumann, J. and Ritter, M., "Steady and unsteady aeroelastic simulations of the HIRENASD wind tunnel experiment," Tech. Rep. IFASD2009-132, Seattle, 2009, International Forum on Aeroelasticity and Structural Dynamics.

${ }^{26}$ Neumann, J., F., N., and Voss, R., “Aeroelastic analysis by coupled non-linear time domain simulation,” Rto report, 2008, RTO-MP_AVT154.

${ }^{27}$ Mavriplis, D., Yang, Z., Long, M., and Sitaraman, J., "Results using NSU3D for the first aeroelastic prediction workshop," AIAA-2013-0786, 51st AIAA Aerospace Sciences Meeting, Grapevine, TX, Jan 2013.

${ }^{28}$ Raveh, D., Yossef, Y., and Levy, Y., "Flow simulations for the first aeroelastic prediction workshop using the EZNSS Code," AIAA-20130787, 51st AIAA Aerospace Sciences Meeting, Grapevine, TX, Jan 2013.

${ }^{29}$ Chwalowski, P., Heeg, J., Wieseman, C., and J.P., F., "FUN3D analyses in support of the first aeroelastic prediction workshop," AIAA paper 2013-0784, 51st AIAA Aerospace Sciences Meeting, Grapevine, TX, Jan 2013.

${ }^{30}$ Nikbay, M. and Acar, P., "Steady and Unsteady Aeroelastic Computations of HiReNASD Wing for Low and High Reynolds Numbers," AIAA-2013-1800, 2013, 54th AIAA/ASME/ASCE/AHS/ASC Structures, Structural Dynamics, and Materials Conference, Boston, Massachusetts, Jan. 8-11, 2013, doi:10.2514/6.2013-1800.

${ }^{31}$ Edwards, J., "Technical evaluation report on 1991 specialists' meeting on transonic unsteady aerodynamics and aeroelasticity," AGARD Paper CP-507, Jan. 1991, AGARD conference proceedings.

${ }^{32}$ Bartels, R. and Sayma, A., "Computational aeroelastic modelling of airframes and turbomachinery: progress and challenges," AIAA Paper 2009-1360, Jan. 2009, Philosophical Transactions of the Royal Society, doi: 10.1098/rsta.2007.2018.

${ }^{33}$ Green, B., Czerwiec, R., Cureton, C., Lillian, C., Kernazhitskiy, S., Eymann, T., Torres, J., Bergeron, K., and Decker, R., "Evaluation of flow solver accuracy using five simple unsteady validation cases," AIAA Paper 2011-29, Jan. 2009.

34"https://c3.nasa.gov/dashlink/projects/47/", January 2013.

${ }^{35} \mathrm{Heeg}$, J., Chwalowski, P., Florance, J., Wieseman, C., Schuster, D., and Perry, B., I., "Overview of the Aeroelastic Prediction Workshop," AIAA-2013-0783, 51st AIAA Aerospace Sciences Meeting, Grapevine, TX, Jan 2013.

${ }^{36}$ Heeg, J., Chwalowski, P., Schuster, D. M., and Dalenbring, M., "Overview and Lessons Learned from the Aeroelastic Prediction Workshop," AIAA-2013-1798, 54th AIAA/ASME/ASCE/AHS/ASC Structures, Structural Dynamics, and Materials Conference, Boston, Massachusetts, Jan. 8-11, 2013.

${ }^{37}$ Heeg, J., Chwalowski, P., Schuster, D., Dalenbring, M., Jirasek, A., Taylor, P., Mavriplis, D., Boucke, A., Ballmann, J., and Smith, M., "Overview and lessons learned from the Aeroelastic Prediction Workshop," IFASD-2013-1a, Bristol, UK, 2013.

${ }^{38}$ Heeg, J. et al., "Plans for an Aeroelastic Prediction Workshop," Tech. Rep. IFASD-2011-110, June 2011, Paris.

${ }^{39}$ Spalart, P. R. and Allmaras, S. R., “A One-Equation Turbulence Model for Aerodynamic Flows,” La Recherche Aerospatiale, No. 1, 1994, pp 5-21.

${ }^{40}$ Menter, F., "Two-Equation Eddy-Viscosity Turbulence Models for Engineering Applications," AIAA Journal, Vol. 32, No. 8, 1994, pp 1598-1605.

${ }^{41}$ Albano, E. and Rodden, W., "A Doublet-Lattice Method for Calculating Lift Distributions on Oscillating Surfaces in Subsonic Flows," AIAA Journal, Vol. 7, No. 2, 1969, pp 279-285.

${ }^{42}$ Chen, P. and Liu, D., “A Harmonic Gradient Mehtod for Unsteady Supersonic Flow Calculations," Journal of Aircraft, Vol. 22, No. 15, May, 1985, pp 371-379.

${ }^{43}$ Bartels, R., "Flow and turbulence modeling and computation of shock buffet onset for conventional and supercritical airfoils," NASA TP 1998-206908, Feb. 1998.

${ }^{44}$ Liggett, N. and Smith, M., "Temporal convergence criteria for time-accurate viscous simulations," Computers and Fluids, Vol. 66, pp. 140156, 2012. doi: 10.1016/j.compfluid.2012.06.010.

${ }^{45}$ Dalenbring, M., Jirasek, A., Chwalowski, P., and Heeg, J., "Initial Investigation of the BSCW Configuration using Hybrid RANS-LES modeling," Tech. rep., 2013, AIAA-2013-1801, 54th AIAA/ASME/ASCE/AHS/ASC Structures, Structural Dynamics, and Materials Conference, Boston, Massachusetts, Jan. 8-11, 2013.

${ }^{46}$ Schuster, D., Chwalowski, P., Heeg, J., and Wieseman, C., "Analysis of test case computations and experiments from the first aeroelastic prediction workshop," AIAA-2013-0788, 51st AIAA Aerospace Sciences Meeting, Grapevine, TX, Jan 2013.

${ }^{47}$ Pranata, B., B., E., van Muijden, J., Heeg, J., Atik, H., and Wieseman, C., "Analysis of First AIAA Aeroelastic Prediction Workshop results of unforced and oscillating HiReNASD wing," IFASD-2013-2b, Bristol, UK, 2013.

${ }^{48}$ Chwalowski, P., Heeg, J., Dalenbring, M., Jirasek, A., Ritter, M., and T., H., "Collaborative HiReNASD analyses to eliminate variations in computational results," IFASD-2013-1d, Bristol, UK, 2013.

${ }^{49} \mathrm{Heeg}$, J. and Wieseman, C., "System identification and uncertainty quantification using orthogonal excitations and the Semi-span SuperSonic Transport ( $\left.\mathrm{S}^{4} \mathrm{~T}\right)$ model," AIAA paper, presented at the 53 ${ }^{\text {rd }}$ AIAA Structural Dynamics and Materials Conference, Honolulu, April 2012.

${ }^{50}$ Cummings, R., Morton, S., and McDaniel, D., "Experiences in accurately predicting time-dependent flows," Progress in Aerospace Sciences, Vol 44, No 4, pp.241-257, 2008.

${ }^{51}$ Morrison, J. H. and Hemsch, M. J., "Statistical Analysis of CFD Solutions from the Thirst AIAA Drag Prediction Workshop," AIAA-2007254, 45th AIAA Aerospace Sciences Meeting and Exhibit, Reno, Nevada, 8-11 Jan., 2007.

${ }^{52}$ Schwamborn, D., Gerhold, T., and Heinrich, R., "The DLR TAU-CODE: Recent Applications in Research and Industry," Technical report at european conference on computational fluid dynamics, ECCOMAS CFD 2006.

${ }^{53} \mathrm{http}: / /$ tau.dlr.de/, Description of the DLR TAU code, March 2012.

${ }^{54}$ Eliasson, P., "EDGE, a Navier-Stokes solver for unstructured grids," Proc. to Finite Volumes for Complex Applications III, ISBN 19039 96341,2002 , pp. 527-534.

${ }^{55}$ Weiss, J. M., Maruszewski, J. P., and Smith, W. A., "Implicit Solution of Preconditioned Navier-Stokes Equations Using Algebraic Multigrid," AIAA Journal, Vol. 37, No. 1, January 1999. 
${ }^{56}$ http://fun3d.larc.nasa.gov, NASA Langley Research Center, November 2010.

${ }^{57}$ Leatham, M., Stokes, S., Shaw, J. A., Cooper, J., Appa, J., and Blaylock, T., “Automatic Mesh Generation for Rapid-Response Navier-Stokes Calculations," Fluids 2000, Conference and Exhibit, 2002.

${ }^{58}$ Bendat, J. and Piersol, A., Random data: Analysis and measurement procedures, John Wiley \& Sons, New York, 1971.

${ }^{59}$ Bendat, J. and Piersol, A., Engineering applications of correlation and spectral analysis, John Wiley \& Sons, New York, 1980.

${ }^{60} \mathrm{https} / / /$ heinrich.lufmech.rwth-aachen.de/en/, Aachen University, June 2010.

${ }^{61}$ Wieseman, C., Chwalowski, P., and Heeg, J., "Structural Dynamics Modeling of HiReNASD in Support of the Aeroelastic Prediction Workshop," AIAA-2013-1801, 2013, 54th AIAA/ASME/ASCE/AHS/ASC Structures, Structural Dynamics, and Materials Conference, Boston, Massachusetts, Jan. 8-11, 2013.

62"http://www.itl.nist.gov/div898/handbook/eda/section3/eda35b.htm", NIST/SEMATECH e-Handbook of Statistical Methods, December 3, 2013.

${ }^{63}$ Boucke, A., "Uncertainties in Measurements and Data Evaluation Concerning Transonic High Reynolds Number Wind Tunnel Tests," IFASD-2013-38c, Bristol, UK, 2013. 